\title{
Correlation of functional status of hip with quality of life of patients with osteonecrosis of femoral head $(\mathrm{ONFH})$
}

\begin{abstract}
Background: The quality of life on patients with ONFH is significantly reduced because of considerable morbidity associated with the disease, especially with bilateral involvement. Although numerous studies describe the clinical characteristic of Osteonecrosis of femoral head, there are very few which have assessed the quality of life of patients with ONFH
\end{abstract}

Purpose: We therefore sought out to determine the: 1) the functional status of the hip as assessed by harries hip score (HHS). (2) The quality of life of the affected patients as assessed by short form 12(SF12) questionnaire.

Patients and Methods: We conducted a study of patients with ONFH from a time period January 2012 till May 2013. 162 newly diagnosed cases of ONFH were included in the study. Established post operative cases were not included in the study.

Results: There were a total 162 patients of ONFH. The mean age of distribution was 34.71 yrs, with $61.1 \%$ cases with bilateral involvement in non traumatic ONFH.ONFH secondary to steroid intake was the leading cause, followed by idiopathic, then secondary ONFH secondary to alcohol consumption. The mean HHS was 80.9 in unilaterally affected patients of ONFH, mean HHS of 72.7 and 80.1 in more involved hip and in less involved hip of Bilaterally ONFH. In patients of ONFH with unilateral involvement, the mean SF12 scores were $49.5 \pm 6.9$ (PCS) and $47.4 \pm 11.5$ (MCS). In patients of ONFH with bilateral involvement, the mean SF12 scores were $46.5 \pm 7$ (PCS) and 39.6 \pm 12.5 (MCS)

Conclusion: Our results confirmed that ONFH is the disease of Middle Ages, which significantly affects the function of the Hip joint which further leads to decreased quality of life because of the morbidity associated with the disease
Volume 7 Issue $3-2017$

\author{
Harsha Vardhan, Ramesh Sen, Vijay Goni, \\ Bhaskar Sarkar, Sameer Aggarwal,Arjun RHH \\ Department of orthopedics, PGIMER, India
}

Correspondence: Harsha Vardhan, Department of Orthopedics, PGIMER, Chandigarh I600I2, India, Tel 9590I2309, Email vardhan86@gmail.com

Received: January 24, 2017 | Published: February 17, 2017

\section{Introduction}

Osteonecrosis is a disease that primarily affects young adults in their thirties and forties, and can lead to a disabling arthritis. ${ }^{1}$ The clinical manifestations vary a lot, while few patients present early, many others get symptoms when Osteonecrosis is in advanced stage. The etiology, natural history and epidemiology of Osteonecrosis of femoral head have also not been fully elucidated. There are very few epidemiological studies in literature which have assessed the risk factors for Osteonecrosis i.e. corticosteroid use, ${ }^{2-4}$ alcohol ${ }^{2-6}$ systemic lupus erythematosus ${ }^{7}$ or organ transplantation ${ }^{8}$ and sickle cell disease ${ }^{9}$ etc.

The problems peculiar to Osteonecrosis of the femur head are relatively younger age of disease onset, variable stage of clinical presentation, significant disability, and a very early need for a surgical intervention, especially the compulsion to go for total hip arthroplasty. ONFH is frequently a bilateral disease, even though patients may be symptomatic on one side. Being a frequent bilateral disease, affecting the middle aged population most often, the disease carries significant morbidity to the patients.

Considering the lack of any epidemiological study in Indian literature and the paucity of epidemiological studies in the world, also just a couple of studies in literature mentioning about the quality of life of patients with ONFH, the current study carries significant importance. In the current study we sought to determine the (1) quality of life of patients with ONFH as assessed by short form 12(SF12) questionnaire. (2) The functional status of the hip as assessed by
Harris hip score (HHS) ${ }^{10}$ and the correlation of SF $12^{11}$ scores with functional status of the hip.

\section{Materials and methods}

This study was conducted in patients with ONFH presenting to us for the first time who were attending to our Services in the department of Orthopaedic surgery of our institution. The patients were seen in OPD and those admitted in wards with the diagnosis of ONFH were enrolled after getting informed consent. All patients above the age of 14 years, irrespective of sex were part of the study. 162 new cases were enrolled for the study.

\section{Selection of cases}

The patients presenting in Orthopedics department from 1 January 2012 till June 2013 with the diagnosis of ON of femoral head were included. Detailed questionnaire were filled after getting information from the patients regarding all possible etiological factors including

A. Corticosteroid treatment- questions were asked about the need for steroid intake, dosage of treatment, duration of treatment

B. Alcohol intake- Amount of alcohol intake, duration was noted

C. Pregnancy

D. Fracture neck of femur and Traumatic dislocation of the hip details about the incident and the duration between the injury and the surgical procedure were asked for 
E. To establish the diagnosis, specific diagnostic work up will be done on clinical suspicion:

i. I.e. gaucher's disease- Serum acid phosphatase estimation

ii. Sickle cell disease- Hemoglobin electrophoresis

iii. Coagulopathies- screening done with PT, PTI, APTT, and later confirmation would be done with estimation of individual clotting factors, Protein C \& S levels

iv. Disturbances of lipid metabolism- lipid profile of the patients would be done

v. Systemic lupus erythematosus-Anti phospholipids antibody detection would be done

vi. HIV infection- HIV Elisa test was done to confirm the diagnosis

vii. Gout- serum uric acid were estimated

F. Idiopathic- if the patient does not fit into any of the above diagnosis then the cause was deemed to be idiopathic in nature

\section{Clinical evaluation}

The patient were examined particularly for any pain, hip deformity and tenderness of hip joints, range of motion, lower extremity muscle group strength (abduction/adduction and flexion/extension of hip), gait and Trendelenburg test and the observations were recorded in the Performa . Harris Hip score was used to records the functional status of the hip at time of presentation.

\section{Radiological evaluation}

The diagnosis was made by obtaining plain Anteroposterior (AP) and lateral radiographs, MRI. The diagnosis of ON of femoral head was based on the revised criteria proposed by Research Committee on Idiopathic Osteonecrosis of femoral head in Japan. ${ }^{12}$ Criteria composed of:

a. Collapse of femoral head including crescent sign, without joint space narrowing on radiographic images

b. Demarcating sclerosis in femoral head without joint space narrowing or acetabular abnormality

c. "Cold in hot" on bone scans

d. Low intensity band on T1- weighted MRI (band like pattern) and

e. Trabecular and marrow necrosis on histopahtological examination.

The patient would be diagnosed as Osteonecrosis if the patient fulfilled 2 of 5 findings and did not have bone tumors or dysplasia. Staging of Osteonecrosis was done based on $\mathrm{ARCO}^{13}$ classification. ARCO's (Association Research Circulation Osseous) international classification of Osteonecrosis has been accepted as a standard protocol for clinical research. Where ever surgical intervention was planned for, biopsy of the femoral head was taken, histopahtological report was considered to be diagnostic. If the femoral head is found to be collapsed on plain radiographs, then the diagnosis will be based only on the radiographic findings. Otherwise, the results of MRI or scintigraphy will be reviewed.

\section{SF-I 2 performa}

After appropriate documentations and requisite information, the short form SF-12 will be used for assessment as has been found to be quick and reliable as is comparable with its longer version, the SF-36, for measuring health related quality of life of patients with various diseases. Staging of the patients was done by the main consultant (RKS), while examination of the patients (HHS) and quality of life scores were recorded by junior orthopedician (HV) to avoid bias.

\section{Results}

There were 162 total numbers of patients of ONFH seeking medical care in our hospital. Among the 162 patients, the mean age of the patients in our study was $35.81 \mathrm{yrs} \pm 10.74 \mathrm{yrs}$ (range $14-70$ yrs). The gender ration was 6.14 with a total of $86.4 \%$ of the patients in our study were males and the rest 13.6 were females. In present series, about $51.2 \%$ of patients had bilateral involvement and if posttraumatic ONFH cases were excluded, this incidence increases to $60.1 \%$ bilateral involvement. In the current study $23.9 \%$ of the total number of patients was idiopathic, where there were no identifiable causes were found. Even in the current study, non traumatic ONFH secondary to steroid intake was accounted for $39.1 \%$. The mean daily intake of prednisolone in unilaterally affected patients was $28 \mathrm{mg}(70 \%$ of patients had consumed $>2 \mathrm{gm}$ prednisolone intake in 2 months) and with bilateral involvement the mean intake was $28.8 \mathrm{mg}$ per day $(86 \%$ of patients had consumed $>2 \mathrm{gm}$ prednisolone intake in 2 months). In our study, out of the total of 167 new patients, 81 patients had unilateral involvement of Osteonecrosis, out of which $4(2.4 \%)$ patients were in stage $1,39(48.1 \%)$ patients were in stage $2,26(32.1 \%)$ patients were in stage 3 and $12(14.8 \%)$ patients were in stage 4 , and the rest 86 patients had bilateral involvement. The mean HHS was 80.9 in unilaterally affected patients of ONFH, mean HHS of 72.82 in more involved hip of Bilateral ONFH. In patients of ONFH with unilateral involvement,

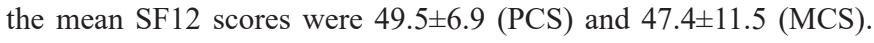
In patients of $\mathrm{ONFH}$ with bilateral involvement, the overall mean SF12 scores were 46.5 \pm 7 (PCS) and 39.6 \pm 12.5 (MCS). Patients with idiopathic and alcohol induced ONFH with bilateral involvement have better physical quality of life when compared to patients with steroid intake (Tables 1-3).

Table I Mean SFI2 scores as per hip functions in cases of unilateral ONFH

\begin{tabular}{lll}
\hline Unilateral Cases & Sf I 2(Mean Pcs) & SfI 2(Mean Mcs) \\
\hline HHS(excellent hip function) & $53.9 \pm 3.0$ & $55.9 \pm 8.0$ \\
HHS(good hip function) & $50.6 \pm 6.4$ & $48.4 \pm 9.9$ \\
HHS( fair hip function) & $49.2 \pm 6.4$ & $42.8 \pm 11.1$ \\
HHS( poor hip function) & $43.4 \pm 7.0$ & $40.3 \pm 11.1$ \\
\hline
\end{tabular}

Table 2 Mean SFI 2 scores as per hip functions in cases of bilateral ONFH

\begin{tabular}{lll}
\hline Bilateral Cases & SfI 2(Mean Pcs) & SfI 2(Mean Mcs) \\
\hline HHS(excellent hip function) & $53.2 \pm 3.3$ & $49.6 \pm 12.0$ \\
HHS(good hip function) & $47.5 \pm 7.2$ & $51.1 \pm 11.2$ \\
HHS( fair hip function) & $49.6 \pm 4.6$ & $35.8 \pm 7.3$ \\
HHS( poor hip function) & $42.2 \pm 6.4$ & $37.3 \pm 12.4$ \\
\hline
\end{tabular}

Table 3 Analysis of Etiology with SFI2 (PCS) in bilateral patients

\begin{tabular}{|c|c|c|c|c|}
\hline Aetiology & $\begin{array}{l}\text { SFI } 2 \text { PCS } \\
<40\end{array}$ & $\begin{array}{l}\text { SF I } 2 \text { PCS } \\
40-50\end{array}$ & $\begin{array}{l}\text { SFI } 2 \text { PCS } \\
>50\end{array}$ & Total \\
\hline Idiopathic & $\mathrm{I}(\mathrm{I} 0 \%)$ & $5(\mid 3.2 \%)$ & $7(25.9 \%)$ & $13(17.3 \%)$ \\
\hline alcohol & $\mathrm{I}(10 \%)$ & $9(23.7 \%)$ & $13(48.1 \%)$ & $23(30.7 \%)$ \\
\hline Steroids & $7(70 \%)$ & $24(63.1 \%)$ & $6(22.2 \%)$ & $37(49.3 \%)$ \\
\hline Trauma & $\mathrm{I}(\mathrm{I0 \%})$ & 0 & I (3.7\%) & $2(2.7 \%)$ \\
\hline $\begin{array}{l}\text { Total } \\
\text { P value }< \\
0.05\end{array}$ & 10 & 38 & 27 & 75 \\
\hline
\end{tabular}

\section{Statistical analysis}

The statistical analysis was carried out using Statistical Package for Social Sciences (SPSS Inc., Chicago, IL, version 17.0 for 
Windows). All quantitative variables were estimated using measures of central tendencies (mean, median) and measures of dispersion (standard deviation and standard error). Normality of data was checked by measures of skewness and Kolmogorov Smirnov tests of normality. For normally distributed data, means were compared using student's t-test for two groups. For skewed data Mann - Whitney test was applied. For time related comparison Paired t-test or ANOVA test was applied as and when there was a need. Qualitative or categorical variables were described as frequencies and proportions. Proportions were compared using Chi- square or Fisher's exact test whichever was applicable. To see correlations for different variables, Pearson Correlations were calculated. All statistical tests were two-sided and performed at a significance level of $\alpha=.05$.

\section{Discussion}

The primary objective of the study was to assess the quality of life of patients with ONFH. We have found a few nationwide epidemiological studies related to Osteonecrosis of femoral head. All of these studies were retrospective studies. Cooper et al. ${ }^{14}$ conducted a case-control study of 601 patients with ONFH and examined its incidence, patient characteristics, and selected potential risk factors using two health record databases in the UK. Fukushima et al. ${ }^{15}$ conducted a nationwide survey in Japan where the information of 1502 diagnosed patients from department of Orthopedics of two hospitals in Japan was collected to determine the distribution of the age and gender of the patients, potential causative factors, severity of the disease, and operative procedures performed. Kang et al. ${ }^{16}$ in Korea analyzed 382 patients with ONFH to estimate its prevalence and to study the disease characteristics. All these studies were done to see the burden of the disease in their respective countries.

There is no other, prospective study in literature on the epidemiology of this disease. One of the reasons could be that the number of patients with this disease is likely to be less in a single hospital setting. Either such a study has to be for prolonged period of time or needs be a multicentre study. In our institutions a lot of such cases are referred from other hospitals for specialized treatment available here for this disease, hence this prospective study was considered possible.

The mean age of 162 patients in our study was 35.81 yrs \pm $10.74 \mathrm{yrs}$ (range 14-70 yrs). Most patients affected were in the $3 \mathrm{rd}$ and 4th decade of life indicating that ONFH is primarily a disease of middle aged adults. As per Fukushima et al. ${ }^{15}$ the peak age of distribution in males was in 40's and in females in their 30's. In a study by Kang et al. ${ }^{10}$ the peak age group was slightly higher i.e. 40 $59 \mathrm{yrs}$. The mean age of patients presenting with ONFH in a series by Sugano et al. ${ }^{13}$ was about 44 years. Thus the age profile in present series is not very different from these other studies except for Cooper et al. ${ }^{14}$ who reported the mean age of $57.5 \mathrm{yrs} \pm 18.9$ in their patients. This difference in the age could be due to their inclusion of patients of $\mathrm{ON}$ from all other sites also in addition to ONFH.

A total of $86.4 \%$ of the patients in our study were males and the rest 13.6 were females. According to Kang et al. ${ }^{16} 77.76 \%$ were males the figure which is not very different from our series. But this difference seems to be more significant while comparing with the series of Fukushima et al. ${ }^{15}$ with only $58.28 \%$ males in their study. In the study by Cooper et al. ${ }^{14}$ this prevalence dips down to $47 \%$ males. No specific cause could be elucidated for such a differences in these studies.

In present series, about $51.2 \%$ of patients had bilateral involvement and if post-traumatic ONFH cases were excluded, this incidence increases to $60.1 \%$ bilateral involvement. As per the study conducted by Sugano et al, bilateral involvement of ONFH comprised about
$67.3 \%$ which is comparable to the generally accepted view. Kang et al. ${ }^{16}$ has however found this bilateral involvement to be only $37 \%$ of patients; this discrepancy was due to non inclusion of the asymptomatic contralateral hip.

In the current study, non traumatic ONFH secondary to steroid intake was accounted for $39.1 \%$. The mean daily intake of prednisolone in unilaterally affected patients was $28 \mathrm{mg}(70 \%$ of patients had consumed $>2 \mathrm{gm}$ prednisolone intake in 2 months) and with bilateral involvement the mean intake was $28.8 \mathrm{mg}$ per day $(86 \%$ of patients had consumed $>2 \mathrm{gm}$ prednisolone intake in 2 months). Fukushima et all1 had defined steroid induced ONFH by a history of taking $1800 \mathrm{mg}$ prednisolone or an equivalent over 4 weeks or by a history of continuous corticosteroid medication for at least 2 months. In the study of Fukushima et al. ${ }^{15}$ systemic steroid administration (steroid induced) accounted for $51 \%$, Thus there is not much difference in this profile. But this is in contrast with the study by Kang et al. ${ }^{16}$ where only $14.6 \%$ were related to steroid intake. Although these rates were comparable to those reported previously in Korea, ${ }^{16}$ they are quite different from those reported from other countries. ${ }^{16}$

In the study of Fukushima et al. ${ }^{15}$ the habitual use of alcohol accounted for $31 \%$. Thus there is not much difference in the etiologic profile secondary to Alcohol intake. In the current study $23.9 \%$ of the total number of patients was idiopathic, where there were no identifiable causes were found. In the study of Fukushima et al. ${ }^{11}$ idiopathic ONFH accounted for $15 \%$ of total cases. Thus there is not much difference in the distribution of this etiological profile.

Harris hip score was used to assess the functional status of the hip and the scores indicating the hip functions as described by Harris $\mathrm{WH}^{10}$ with HHS $<70$ indicating poor functional status of hip, HHS7079 indicating fair hip functional status, HHS80-89 indicating good functional status of the hip and $>90$ indicating excellent hip functions.

The functional part of HHS varies in cases with bilateral involvement. In patients with bilateral ONFH, some patients have complaints with one side and asymptomatic on the other, some patients have one hip being more symptomatic than the other, or and in some patients both hips are equally affected. Hence in the current study, in patients with bilateral involvement the hip with the least score was taken for analysis.

The mean HHS was 80.9 in unilaterally affected patients of ONFH, mean HHS of 72.7 more involved hip of Bilateral ONFH.

It is a well proven fact that the quality of life gets affected with many of the diseases. There are a number of scales to assess the quality of life of patients. In the current study the Short Form 12 score was used to assess the quality of life. Short Form 12(SF12) score has been used for quick and reliable assessment of quality of life of patients. The SF12 score takes into account both the physical (PCS) and the mental component (MCS) to assess the quality of life.

When looking at various studies published on Epidemiology of ONFH in Pubmed, we found only two studies which had used SF12 score to analyze the quality of life of patient with ONFH. In the study SF12 was used to measure and compare the outcome of ONFH following hip resurfacing arthroplasty and Total Hip Arthroplasty. A total of 381 patients were studied by Whitehouse et al. ${ }^{17}$ As Hip replacement were being done in the terminal stages of ONFH it would not be suitable for comparison for the current study.

The SF12 scores(Both PCS and MCS) were analyzed for correlation among the sexes, etiologic factors, involvement of the hip, stage of the disease and the functional status of the hip being measured with HHS. 
In patients with bilateral involvement, patients with idiopathic and alcohol induced ONFH had a better physical quality of life when compared to patients with steroid intake.

In patients with unilateral involvement of hip, with good hip functions had a better quality of life when compared with patients with $(\mathrm{HHS}<80$ ) fair and poor functional status of hip.

In patients with bilateral involvement, with HHS $>80$ in the more affected hip have better quality of life (both PCS \& MCS) when compared with patients with $<80$ HHS.

\section{Acknowledgments}

None.

\section{Conflicts of interest}

None.

\section{References}

1. Jones CL, Mont MA, Johnson AJ, et al. Osteonecrosis of the Hip in Adults. Clinic Rev Bone Miner Metab. 2011;9(1):13-22.

2. Boskey AL, Raggio CL, Bullough PG, et al. Changes in the bone tissue lipids in persons with steroid and alcohol induced Osteonecrosis. Clin Orthop Relat Res. 1983;172:289-295.

3. Koo KH1, Kim R, Kim YS, et al. Risk period for developing Osteonecrosis of the femoral head in patients on steroid treatment. Clin Rheumatol. 2002;21(4):299-303.

4. Pavelka K. Osteonecrosis. Baillieres Best Pract Res Clin Rheumatol. 2000;14(2):399-414.

5. Shibata A, Fukuda K, Inoue A, et al. Flushing pattern and idiopathic avascular necrosis of the femoral head. J Epidemiol. 1996;6(1):37-43.

6. Matsuo K, Hirohata T, Sugioka Y, et al. Influence of alcohol intake, cigarette smoking, and occupational status on idiopathic Osteonecrosis of the femoral head. Clin Orthop Relat Res. 1988;234:115-123.
7. Gorshtein A, Levy Y. Orthopedic Involvement in Antiphospholipid Syndrome. Clinic Rev Allerg Immunol. 2007;32(2):167-171.

8. Metselaar HJ, van Seenberge EJ, Bijnen AB, et al. Incidence of Osteonecrosis after renal transplantation. Acta Orthop Scand. 1985;56(5):413-415.

9. Babhulkar SS. Avascular Necrosis of Femoral Head in Sickle Cell Haemoglobinopathies. Indian J Orthop. 1981;15(2):162-165.

10. Harris WH. Traumatic arthritis of the hip after dislocation and acetabular fractures: Treatment by mold arthroplasty. An end-result study using a new method of result evaluation. J Bone Joint Surg Am. 1969;51(4):737-755.

11. Dempster M, Donnelly M. A comparative analysis of the SF-12 and SF-36 among ischemic heart disease patients. J Health Psychol. 2001; 6(6):707-711.

12. Schmitt-Sody M, Kirchhoff C, Mayer W, et al. Avascular necrosis of the femoral head: inter- and intraobserver variations of Ficat and ARCO classifications. Int Orthop. 2008;32(3):283-287.

13. Sugano N, Kubo T, Takaoka K, et al. Diagnostic criteria for non traumatic Osteonecrosis of femoral head. J Bone Joint Surg Br. 1999;81(4):590-595.

14. Cooper C, Steinbuch M, Stevenson R, et al. The epidemiology of Osteonecrosis: findings from the GPRD and THIN databases in the UK. Osteoporos Int. 2010;21(4):569-577.

15. Fukushima W, Fujioka M, Kubo T, et al. Nationwide Epidemiologic Survey of Idiopathic Osteonecrosis of the Femoral Head. Clin Orthop Relat Res. 2010;468(10):2715-2724.

16. Kang JS, Park S, Song JH, et al. Prevalence of Osteonecrosis of the Femoral Head: A Nationwide Epidemiologic Analysis in Korea. $J$ Arthroplasty. 2009;24(8):1178-1183.

17. Whitehouse MR, Aquilina AL, Patel S, et al. Survivorship Patient reported outcome and satisfaction following resurfacing and total hip arthroplasty. J Arthroplasty. 201328(5):842-848. 